\title{
Zehn Königsurkunden für Reichsburgmannen des hessischen und pfälzischen Gebiets, 127\%-1323.
}

\author{
Mitgeteilt von
}

\section{E. Sch a u s,}

Acht der folgenden Urkunden sind überliefert in einem kleinen bisher unbeachteten Kopiar des Wiesbadener Staatsarchivs, das aus dem Nachlass des wassauischen Geschichtsforschers C. D. Vogel stammt und in einer Aufschrift von ihm als Geschenk des früheren speyerischen Domkapitulars Freiherrn Schütz zu Hclzhauseu in Kamberg bezeichnet wird. Es enthält auf 20 Blättern 38 oder unter Zurechuung eines inserirten Stückes 39 Urkunden in Abschriften, die von eiuer gleichmässigen Hand des 17. Jahrhunderts herrühren, aber nicht fehlerfrei sind. Nach manchen Bemerkungen über die Besiegelung scheinen die Vorlagen zum Teile wenigstens Originale gewesen zu sein. Während auf den übrigen Inhalt hier nicht eingegangen werden soll, sei nur erwähnt, dass Blatt 14 einen Text der Urkunde König Albrechts für Peter von Lörzweiler (bei Oppenheim), Böhmer Reg. Alb. 449, bietet, der riehtig: a nobis et imperio hat statt des sinnlosen in bei Würdtwein Diplomataria Magunt. 1, 101, der ferner im Datum: XVIII kal. sept. aufweist, was dem VIII kal, des Druckes auch vorzuziehen sein wird, da derselbe Empfänger am 15. August 1303 eine andere Urkunde B. 446 vom König erhält.

Nr. 2 und 7 haben ganz moderne Abschriften aus dem Düsseldorfer Staatsarchiv zur Vorlage. Das „Kopiar der Edelherrn und Grafen von Zweibrücken aus dem 15. Jahrhundert", nach dem sie 1871 kopirt wurden, scheint inzwischen verschollen zu sein. Damals 
war die Handschrift, die der Habelscheu Sammlung auf Schloss Miltenberg angehört hat, von dem Archivdirektor Baur in Darmstadt leihweise nach Düsseldorf mitgeteilt worden; diesem Umstind ist die Kenntnis der hiemit vorgelegten T'exte zu danken.

Die Urkunden insgesamt verdienen vielleicht die Wiedergabe, weii sie einen Beitrag zur Geschichte des Reichsguts und der Reichsburgmannschaft in der Pfalz und in Rheinhessen bieten; sie ergänzen die reichlich vorhandenen Quellen über diesen Gegenstand, der wohl einer zusammenfassenden Bearbeitung wert wäre ${ }^{1}$ ).

1.

König Rudolf erlaubt seinem Schultheissen Werner von Oppenheim zur Verbesserung seines Burglehns vier Mühlen in den Rhein zu bauen. 1277 April 25, Wien.

Rudolfus dei gratia Romanorum rex semper augustus universis sacri Romani imperii fidelibus presentes literas inspecturis volımus notum esse, quod nos dilecto fideli nostro Wernhero sculteto de Oppenheim in augmentum feodi sui castreusis apud Oppenheim quatuor aquas molendinarias in alveo Reni ibidem, ubi sibi magis oportunum viderit, de regalis liberalitatis munificentia duximus concedendas, dautes sibi tenore presentium plenam potestatem totidem molendina in aquis eisdem locandi iuxta sue beneplacitum voluntatis. In cuius concessionis testimonium presens scriptum exinde conscribi et maiestatis nostre sigillo iussimus roborari. Datum Wienne, VII. kalend. maii, indictione $\mathrm{V}$, anno domini MCCLXXVII, regni vero nostri anno quarto.

Kopiar C Nr. 2, Bl. 18 im Wiesbadener Staatsarchiv. Die Urkunde ist eingerückt in der Bestätigung des Königs Adolf von 1294 Januar 24, s. unten Nr. 5. Sie reiht sich der vom selben Tag Böhmer-Redlich 747 an.

2.

König Rudolf nimmt Merbodo von Wilenstein zum Reichsburgmann in Kaiserslautern an.

1287 Dezember 19, Speyer.

Nos Rudolphus dei gratia Romanorum rex senper augustus ad universorum sacri imperii Romani fidelium notitiam volumus pervenire,

1) Vgl. übrigens jetzt die kürzlich erschienene Arbeit von Hans Niese Die Verwaltung des Reichsgutes im 13. Jahrhundert (Innsbruck 1905) S. 222 ff., auch H. Schreibmüller Die Landvogtei im Speiergau (Progr. d. Gymnasiums Kaiserslautern 1905). Eine Burglehensurkunde K. Rudolfs von 1277 Febr. j betr. Schloss Nicastel beim Trifels veröffentlichte jüngst Redlich in dieser Zeitschrift 25, 327 Anm. 2. 
quod nos iuspecta fidei et puritatis constantia, qua strenuus vir Merbodo de Wylenstein dilectus noster fidelis erga nos incessanter enituit, in castro nostro apud Lutream nobis et imperio in castellanum duximus conquirendum et pro eo sibi centum libras Hallensium promittimus nos daturos, pro quibus sibi decem librarum Hallensium redditus percipiendos de officio nostro, quod dicitur bo̊dilampt, ita videlicet quod singulis annis quinque libras et in festo beati Remigii alias quinque percipiat, duximus obligandos, tenendos quousque sibi per nos vel successores nostros in imperio dicte centum libre plenarie fuerint persolute; quibus solutis ipse Merbodo de dictis centum libris predia comparabit et ipsa predia in dicto castro Lutrensi deserviens nomine castrensis feodi in ipso castro a festo beati Martini usque ad festum pasche omnibus annis ${ }^{1}$ ) residebit. Presentium testimonio litterarum. Datum Spire, XIIII. kalendis ianuarii, indictione XV, anno domini millesimo ducentesimo octogesimo septimo, regni anno quinto decimo.

Abschrift aus dem Jahr 1871 im Düsseldorfer Staatsarchiv, Kopiar B. $177^{\text {b }}$ Bl. 37 ; die Vorlage war ein Zweibrücker Kopiar des 15 . Jahrhunderts, ebedem in der Habelschen Sammlung zu Miltenberg. Über die Burg Wilenstein bei Kaiserslautern s. J. G. Lehmann Urkundliche Geschichte der Burgen in der bayerischen Pfalz 5, Kaiserslautern 1866, S. $63 \mathrm{ff}$.

3.

König Rudolf nimmt Merbodo von Breitenborn zum Reichsburgmann in Kaiserslautern an.

1291 April 5, Germersheim.

Nos Rudolffus dei gratia Romanorum rex semper augustus ad universorum sacri imperii Romani fidelium notitiam volumus pervenire, quod nos inspecta fidei et puritatis constantia, qua strenuus vir Merbodo de Breidenburne miles erga nos enituit incessanter, ipsum in castro Lutra nobis et imperio in castellanum duximus conquirendum, et proinde sibi octuaginta libras Hallensiun promittimus nos daturos, pro quibus eidem redditus octo librarum in officio Kebelinburg, quarum quatuor in maio, residuas quatuor in festo Remigii eidem assignari volumus per officiatum nostrum qui pro tempore fuerit, duximus obligandos, tenendos et possidendos tam diu ab ipso et suis heredibus, quousque sibi vel suis heredibus premissis de prefatis octuaginta libris fiat plenarie satisfactum per nos vel nostros in imperio successores. Solutione autem facta huiusmodi ipse ${ }^{2}$ ) aut sui heredes prefatam sum-

1) famulis in der Vorlage.

2) fehlt in der Vorlage. 
mam infra netas officii de Lutra convertent in predia et eadem ${ }^{1}$ ) in castro Lutra nomine castrensis feodi deservire perpetuo tenebuntur. Preterea indulgemus eisdem, ut eo tempore anni quo maluerint per dimidium annum duntaxat in dicto castro facient residentiam personalem. Presentium testimonio litterarum. Datum Germirsheim, nonis aprilis, indictione quarta, anno domini MCCnonagesimo primo, regni vero nostri auno decimo octaro.

Aus gleicher Quelle wie Nr. 1, Bl. 1. Unter dem Text die falsche Siegelangabe: Locus sigilli. Ludovicus dei gratia Romanorum rex semper augustus. Über die auch im Gebiet von Kaiserslautern gelegene Burg des Empfängers, den jetzigen Breitenborner Hof, s. Lehmann Burgen 5, 38. $\mathrm{Zu}$ Kebelnberg $=$ Kübelberg $\mathbf{n}$. Homburg in der Pfalz vgl. Böhmer Acta imperii 434 Nr. 620 und $450 \mathrm{Nr} .642$.

4.

König Rudolf genehmigt die von dem Schultheissen Werner zu Oppenheim vollzogene Vertauschung eines dem Reich gehörigen Weinbergs zu Nierstein gegen 2 andere aus dem Besitz Werners.

$$
1291 \text { April 9. [Speier]. }
$$

Rudolfus dei gratia Romanorum rex semper augustus universis sacri imperii tidelibus gratiam suam et omne bonum. Universitati vestre constare volumus per presentes, quod cum egregius vir Wernherus scultetus de Oppenheim fidelis noster dilectus de vineis suis sitis in terminis ville nostre in Nerstheim iuxta Oppenheim scilicet in Rietburn et Urvar ac vinea curie Mandonis militis ibidem contigua nobis et imperio attinente de consilio scabinorum eiusdem ville fecerit permutationem, nos meliorationem conditionis nostre et imperii evidenter in his perpendentes, maxime cum de consilio dictorum scabinorum factum existat, ipsam permutationem ratam et gratam habentes eidem auctoritatem plenariam impertinur, dantes super hiis has literas sigillo nostre maiestatis regie roboratas. Actum et datum anno domini MCCLXXXX primo, V. idus aprilis, indictione quarta, regni vero nostri anno XVIII.

Aus gleicher Quelle wie Nr. 1, Bl. 19. Der fons Rytborn in der Niersteiner Gemarkung begegnet in einer Urkunde von 1395 s. Baur Hessische Urkunden 5, S. 493 Über den Ritter Moydo, Moudo von Nierstein s. Baur 2, 187, 260; 3, 628. Auf diese Urkunde scheint sich übrigens die Angabe zu beziehen, die sich in einem Verzeichnis der Reifenberger Dokumente von 1594 im Staatsarchiv zu Wiesbaden findet und die bei Sauer, Nassauisches Urkundenbuch I Vorbemerkungen S. XXVI

1) e od em Vorlage. 
Anm. kurz wiedergegeben ist; sie lautet: Confirmatio von keiser Rudolpho einen dausch etlicher gueter zu Nirsthein den schulteissen zu Oppenheim betreffende anno 1290 under gemeltes keyssers gross. insigel. Vgl. unter Nr. 5.

5.

König Adolf bestätigt das Mühlenprivileg seines Vorgängers Rudolf für den Schultheissen Werner von Oppenheim von $127 \%$ April 25. 1294 Januar 22, Frankfurt.

Adolfus dei gratia Romanorum rex semper augustus universis imperii Romani fidelibus presentes literas inspecturis gratiam suam et omne bouum. Inclite recordatiouis Rudolfi Romanorum regis illustris nostri predecessoris literas vidimus et audivimus in hec verba: Es folgt der Wortlaut der Urkunde von 1277 April 25, oben $\mathrm{Nr}$. 1. Nos igitur Adolfus Pomanorum rex predictam concessionem per prefatum Rudolfum regem rite et provide factam approbamus et auctoritate regia confirmamus, dantes has literas in testimonium super eo. Datum Frankenvort, XI kal. febr. indictione septima, anno donini MCC nonagesimo quarto, regni vero nostri anno secundo.

Quelle wie Nr. 1. Im Verzeichnis der Reifenberger Dokumente von 1594, s. bei Nr. 4, heisst es: Item ein Privilegium keisers Adolphi dem schulteissen zu Oppenheim, ein muele in den Rein zu bauhen, anno 1294; s. Sauer a. a. 0. Während in diesen zwei Fällen wenigstens der Text der Urkunden durch unser Kopiar gerettet zu sein scheint, ist mit einer weiteren Angabe des Verzeichnisses leider gar nichts mehr anzufangen; sie lautet: Item vier uralt briflin uber etlichen wein zu Nirsthein under keiser Rudolphi primi \& Adolphi auch eines graven von Falckenstein siglung Wernhero schulteissen zu Oppenheim verlauhen. Über den Verbleib aller dieser Urkunden ist beute auch nicht einmal eine Vermutung mehr möglich.

6.

König Heinrich VII. genehmigt, dass Gerhard der Sohn des verstorbenen Schultheissen Werner von Oppenheim, seiner Frau Elisabeth eine ihm vom Reich verliehene Weingülte als Mitgift anweist.

\section{März 4, Speyer.}

Nos Henricus dei gratia Romanorum rex semper augustus ad universorum sacri imperii fidelium notitiam volumus pervenire, quod strenuum virum Gerhardum filium quondam Wernheri sculteti in Oppinheim ob grata que ut audivimus imperio impendit obsequia et gratiora que nobis et eidem imperio exhibere in futurum poterit, favore benevolo prosequi disponentes, sibi hanc motu liberalitatis regie gratiam duximus faciendam, quod Elizabeth uxori sue legitime redditus 
duarum carratarum vini, quos de vineis nostris et imperii in feodum habere se asserit, de nostro consensu nomine dotis valeat assignare. In cuius rei testimonium has literas nostre maiestatis sigillo iussimus communiri. Datum Spire, quarto nonas martii, anno domini MCCCIX, regni nostri anno primo.

Aus gleicher Quelle wie Nr. 1, Bl, 16.

7.

König Heinrich VII. genehmigt, dass Wirich von Wilenstein, Reichsburgmann zu Kaiserslautern, seiner Frau Hildegard sein Burylehen anweist, bis sie das ihr versprochene Heiratsgut von 100 Pfund Heller ganz empfangen hat.

1310 August 11, Kaiserslautern.

Nos Heinricus dei gratia Romanorum rex semper augustus ad universorun notitiam volumus pervenire, qnod grata et fidelia servicia strenui viri Wirici de Wylensten castrensis nostri in Lutrea exhibita imperio et nobis in posterum exhibenda gratiosius intuentes, ip ius devotis precibus favorabiliter inclinati tenore presentium indulgemus et concedimus eidem, quod feodum castrense quod in castro nostro Lutrensi optinere dinoscitur, Hyldegardi uxori sue legittime pro centum libris Hallensium debitis eidem ratione dotis et promissis per eum licite valeat assiguare tenendum per eandem, donec sibi de centum libris predictis integraliter satisfiat. In cuius rei testimonium presentes litteras 〈conscribi〉 et nostre maiestatis sigillo iussimus communiri. Datum in Lutrea, III. idus augusti, anno domini millesimo trecentesimo decimo, regni vero nostri anno II.

Aus gleicher Quelle wie Nr. 2, Bl. 73 .

8.

König Heinrich VII. nimmt Georg von Randeck zum Burgmann in Oppenheim an.

\section{August 31, in Lager vor Brescia.}

Henricus dei gratia Romanorum rex semper augustus universis sacri Romani imperii fidelibus presentes literas inspecturis gratian suam et omne bonum. Grata et fidelia servitia que vir strenuus Georgius de Randekke dilectus noster fidelis nobis exhibuit et impesio in partibus Italie et exhibere poterit in futurum, merito nos inducunt, ut ipsum favore benevolo prosequamur. Hinc est quod eundem Georgium in nostrum et imperii castrensem apud Oppenheim duximus conquirendum, dantes sibi propter hoc decem et octo libras Hallensium, 
quas sibi et heredibus suis apud iudeos nostros de Oppenheim de stura ${ }^{1}$ ) per eos solvenda in festo beati Martini annis singulis colligendas presentibus deputamus, percipiendas tamdiu et habendas, quousque sibi aut heredibus suis per nos vel nostros in imperio successores centum et octoginta libre Hallensium plenarie fuerint persolute; quibus solutis convertent in predia et ea vel saltem de suo allodio tantum ipse et heredes sui titulo castrensis feodi iuxta morem et consuetudinem aliorum castrensium feodorum, que vulgariter ledik burchlehen dicuntur, deserviendi perpetuo possidebunt ${ }^{2}$ ). In cuius rei testimonium presentes literas maiestatis nostre sigillo iussimus communiri. Datum in castris ante Brixiam, II. kalendas septemb. anno domini Mo trecentesimo undecimo, regni vero nostri auno tertio.

Aus gleicher Quelle wie Nr. 1, Bl, 11. - Die Urkunde ist als ungedruckt erwähnt von Bodmann Rheingauische Alterthümer (Mainz 1819) S. 549. Über Randeck bei Mannweiler an der Alsenz s. Lehmann Burgen $4,212 \mathrm{ff}$.

9.

König Ludwig der Bayer verleiht dem Ritter Wigand von Dienheim ein Haus in der Reichsburg Schwabsburg samt einem Fischteich als Burglehen.

\section{Dezember 15, Oppenheim.}

Nos Ludovicus dei gratia Romanorum rex semper augustus ad universorum notitian volumus pervenire, quod strenuo viro Wigando de Diesheim militi dilecto fideli nostro fidelium servitiorum suorum obtentu domum nostram sitam in castro nostro Swabesburg que cenaculum appellatur necnon piscariam sive lacum situm prope castrum predictum in feodum castrense tenendam et possidendam per eum et heredes suos perpetuo de liberalitate nostra concessimus et tenore presentium concedimus et donamus; ita tamen quod prefatus Wigandus eandem domum cenaculi in locis ubi indiget congrua reparatione reformet et cum nos in dicto castro hospitari contigerit vel heredes nostros ipsam domum nobis et nostris heredibus aperiat et patentem faciat ad hospitandum in ea sicut fuerit oportunum. In cuius rei testimonium presentes literas conscribi et sigillo nostro iussimus communiri. Datum in Oppenheim, XVIII. kal. ianuarii, anno dumini millesimo trecentesimo septimo decimo, regni vero nostri anno quarto.

Aus gleicher Quelle wie Nr. 1, Bl. 15. - Dienheim südlich und Schwabsburg westlich von Oppenheim.

1) stura ist ergänzt; die Vorlage hat eine Lücke.

2) possidendum in der Vorlage. 
10.

König Ludwig der Bayer verleiht dem Emerich von Lewenstein das Burglehen in Oppenheim, das sein verstorbener Schwager Ludolf von Schmidburg bisher besessen hat.

1323 Juli 26, Nürnberg.

Nos Ludovicus dei gratia Romanorum rex semper augustus ad universorun notitiam volumus pervenire, quod strenuo viro Emerico de Leonstein fideli nostro dilecto feodum castrense in Oppenheim, quod quondam Ludolfus de Smideburch sororius suus a nobis et imperio tenuit ad instantian et requisitionem Demudis eiusdem sororis contulimus et conferimus perpetuo possidendum et heredes ipsius sibi succedere volumus in feodo memorato. Ex ea etiam infeudatione predictus Emericus ac heredes sui nobis et imperio tenentur sub fidelitatis homagio deservire. In cuius rei testimonium presentes litteras sigillo maiestatis nostre iussimus communiri. Datum in Nurenberg, VII. kal. augusti, anno domiui MCCC vicesimo tertio, regni vero nostri anno nono.

Aus gleicher Quelle wie $\mathrm{Nr}$. 1, Bl. 3. Mit der Siegelumsehrift unter dem Text Ludovicus dei gratia Ronanorum rex semper augustus. Lewenstein bei Obermoschel, s. Lehmann Burgen 4, 251. Schmidburg im Hahnenbachtal, Rheinprovinz Kr. Simmern. 